\title{
Racial Congruence in Physical Activity Interventions among Older African Americans
}

\author{
Leah Carter*, Callie Hebert, Owen Carmichael, Robert L Newton Jr \\ Pennington Biomedical Research Center, Baton Rouge, Louisiana
}

Article Info

Article Notes

Received: March 5, 2020

Accepted: March 28, 2020

\section{*Correspondence:}

Leah Carter, M.Ed, Project Manager, Pennington Biomedical Research Center, Physical Activity and Ethnic Minority Health Lab, Louisiana, USA, 70808; Telephone No: 225-763-3080; Email: Leah.carter@pbrc.edu.

(c) 2020 Carter $L$. This article is distributed under the terms of the Creative Commons Attribution 4.0 International License.
In a recent article, Jedrziewski and colleagues $^{1}$ presented feasibility data for their African Dance intervention among older community-dwelling African American adults. One of their assertions was that racial congruence among all staff is a necessity for successful exercise trials with older African American participants ${ }^{1}$. The purpose of this commentary is to provide a different perspective on the question of racial congruence, based on our own experience recruiting and retaining older African American adults into a physical activity promotion study.

The efforts of Jedrziewski and colleagues to better enhance and develop research engagement among older African American adults are highly commendable as few studies have sought to intervene exclusively with this population despite the great need. The prevalence of Alzheimer's disease (AD) is high in the general population ${ }^{2}$. Studies have shown that the incidence of $\mathrm{AD}$ among African Americans may be as much as twice as high compared to non-Hispanic white Americans ${ }^{3,4}$. For example, US census standardized estimates of AD incidence was $4 \%$ in African Americans and $2 \%$ in non-Hispanic white Americans according to the Chicago Health and Aging Project ${ }^{5}$. The disparities in cognitive outcomes that African Americans face are driven by a variety of early life risk factors including environment, lifestyle, and metabolic and cardiovascular chronic disease ${ }^{6}$. Yet, African Americans participate in aging research less frequently than non-Hispanic white Americans ${ }^{7}$. Therefore, there are now efforts underway to identify strategies to both recruit and retain African Americans into AD-related research studies ${ }^{8}$. Many studies outside of the dementia field have documented successful recruitment strategies for African Americans, including mass media and grass-roots methods ${ }^{9-11}$. It has also been noted that utilizing African American recruiters ${ }^{12,13}$ and outreach coordinators ${ }^{14}$ and receiving an endorsement from community leaders are key components ${ }^{15}$.

Like Jedrziewski and colleagues ${ }^{1}$, our group is specifically concerned with recruiting older African American adults into AD-related physical activity promotion studies. Our research is conducted in Baton Rouge, Louisiana. Whereas $46 \%$ of older non-Hispanic white Americans report 150 minutes per week of moderate to vigorous physical activity, only $33 \%$ of older African Americans meet this criteria ${ }^{16}$. These data suggest that older African Americans are especially well poised to benefit from physical activity promotion programs. Therefore, we designed the Program for African American Cognition and Exercise (PAACE), a randomized 
clinical trial designed to assess the effectiveness of a physical activity intervention on cognitive functioning in older African Americans. We recruited 56 older African American adults aged 65 to 85 years. PAACE participants were randomized into either a physical activity group (PAG) or a successful aging group (SAG) for 12 weeks. Outcome measures included physical function, cognitive function, blood pressure, cardiometabolic blood biomarkers, quality of life, and mood. Participants in the SAG attended twelve, 30-60 minute group sessions at Pennington Biomedical. The group sessions were structured to foster interactive discussion on topics including healthy eating, living wills, and dementia awareness. Participants in the PAG attended two supervised physical activity sessions per week, and participants were instructed to engage in 2-3 days of physical activity at home ${ }^{17}$. The program was informed by older African Americans and took place in a local YMCA facility.

Each potential participant's first exposure to the study usually came through community-based outreach efforts or via the study website. We utilized community-based outreach methods previously shown to be effective by the investigators, including presentations at churches, tabling at health fairs, and attendance at other community events specifically targeting African Americans ${ }^{18-20}$. If interested, a phone screen, orientation session, and baseline clinic visit would be required before being randomized. Community-based outreach efforts were mainly conducted by the institution's outreach coordinator, supplemented by a research assistant (LC) and one of the Co-PIs (RN), all of whom were African Americans aged 35-55. Another recruiter, staff who completed the face-to-face orientations and clinic visits, and the study coordinator were nonHispanic white Americans. Furthermore, the leaders for the PAG were either non-Hispanic white Americans or Hispanic Americans aged 25-30. The leader of the SAG was a 61-year old African American woman who was a Registered Dietitian and Certified Diabetes Educator. All intervention staff were employees of Pennington Biomedical and trained in Good Clinical Practices and Cultural Competency. Our study achieved 93\% and 87\% attendance in the PAG and the SAG, respectively. Overall, $92 \%$ of participants completed both the baseline and 12 week assessments.

There are notable similarities and differences between the studies that have implications for cultural congruence. Both studies recruited cognitively normal older African American adults, were physical activity interventions, and were short-term. However, there was also one important difference: the Jedrziewski study ${ }^{1}$ was culturally-tailored, in that the physical activity arm engaged in African dance and the control arm received education in African American history. Racial concordance may be especially important for such a culturally-tailored intervention, to address both surface and deep structure ${ }^{20}$.

Like Jedrziewski and colleagues ${ }^{1}$, most studies targeting African Americans utilize racially concordant recruitment staff when making the first contact with potential participants. This practice likely helps establish rapport. However, staffing constraints may make racial concordance across all aspects of participant's experience difficult to achieve. Our experiences show that racial discordance can still result in successful recruitment and high attendance. Therefore we draw different conclusions from that of Jedrziewski and colleagues ${ }^{1}$-race concordance was far from essential to successfully complete a relatively large physical activity trial in older African Americans.

\section{Acknowledgments}

This study was funded by a grant from the BrightFocus Foundation.

None of the authors have conflicts of interest and all authors participated in the study and have seen and approved this manuscript prior to submission.

\section{References}

1. Jedrziewski MK, Meekins D, Gorka SA, et al. Feasibility of a Randomized Controlled Trial to Test the Impact of African Dance on Cognitive Function and Risk of Dementia: the REACT! Study. Journal of mental health \& clinical psychology. 2018; 2(1): 12-13.

2. 2014 Alzheimer's disease facts and figures. Alzheimers Dement. 2014; 10(2): e47-92.

3. Gurland BJ, Wilder DE, Lantigua R, et al. Rates of dementia in three ethnoracial groups. Int J Geriatr Psychiatry. 1999; 14(6): 481-493.

4. Tang MX, Cross P, Andrews $\mathrm{H}$, et al. Incidence of AD in AfricanAmericans, Caribbean Hispanics, and Caucasians in northern Manhattan. Neurology. 2001; 56(1): 49-56.

5. Rajan KB, Weuve J, Barnes LL, et al. Prevalence and incidence of clinically diagnosed Alzheimer's disease dementia from 1994 to 2012 in a population study. Alzheimers Dement. 2019; 15(1): 1-7.

6. Cermakova P, Muller M, Armstrong AC, et al. Subclinical Cardiac Dysfunction and Brain Health in Midlife: CARDIA (Coronary Artery Risk Development in Young Adults) Brain Magnetic Resonance Imaging Substudy. Journal of the American Heart Association. 2017; 6(12): 006750.

7. Gluck, Mark A, Ashlee Shaw, et al. "Recruiting older African Americans to brain health and aging research through community engagement." Generations 42. 2018; 2: 78-82.

8. Barnes LL, Bennett DA. Alzheimer's disease in African Americans: risk factors and challenges for the future. Health affairs (Project Hope). 2014; 33(4): 580-586.

9. Brown SD, Lee K, Schoffman DE, et al. Minority recruitment into clinical trials: experimental findings and practical implications. Contemp Clin Trials. 2012; 33(4): 620-623.

10. Andreae SJ, Halanych JH, Cherrington A, et al. Recruitment of a rural, southern, predominantly African-American population into a diabetes self-management trial. Contemp Clin Trials. 2012; 33(3): 499-506.

11. Wilbur J, Buchholz SW, Ingram DM, et al. Effectiveness, efficiency, duration, and costs of recruiting for an African American women's 
lifestyle physical activity program. Res Nurs Health. 2013; 36(5): 487 499.

12. Germino BB, Mishel MH, Alexander GR, et al. Engaging African American breast cancer survivors in an intervention trial: culture, responsiveness and community. J Cancer Surviv. 2011; 5(1): 82-91.

13. Johnson VA, Powell-Young YM, Torres ER, et al. A systematic review of strategies that increase the recruitment and retention of African American adults in genetic and genomic studies. ABNF J. 2011; 22(4): 84-88.

14. King DW, Duello TM, Miranda PY, et al. Strategies for Recruitment of Healthy Premenopausal Women into the African American Nutrition for Life (A NULIFE) Study. J Womens Health (Larchmt). 2010; 19(5): 855-862.

15. Kennedy BM, Harsha DW, Bookman EB, et al. Challenges to recruitment and retention of African Americans in the gene-environment trial of response to dietary interventions (GET READI) for heart health. Health Educ Res. 2011; 26(5): 923-936.

16. Keadle, Sarah Kozey. "Prevalence and trends in physical activity among older adults in the United States: A comparison across three national surveys." Preventive medicine. 2016; 89: 37-43. doi:10.1016/j. ypmed.2016.05.009

17. Sink KM, Espeland MA, Castro CM, et al. Effect of a 24-Month Physical Activity Intervention vs Health Education on Cognitive Outcomes in Sedentary Older Adults: The LIFE RandomizedTrial. Jama. 2015; 314(8): 781-790.

18. Newton RL Jr, Perri MG. A randomized pilot trial of exercise promotion in sedentary African-American adults. Ethn Dis. 2004; 14(4): 548557.

19. Newton RL Jr, Johnson WD, Hendrick C, et al. A randomized controlled exercise training trial on insulin sensitivity in African American men: The ARTIIS study: Major category: study design, statistical design, study protocols. Contemp Clin Trials. 2015; 43: 75-82.

20. Newton RL Jr, Carter LA, Johnson W, et al. A Church-Based Weight Loss Intervention in African American Adults using Text Messages (LEAN Study): Cluster Randomized Controlled Trial. J Med Internet Res. 2018; 20(8): e256. 